PROCEEDINGS OF THE

AMERICAN MATHEMATICAL SOCIETY

Volume 125, Number 4, April 1997, Pages 1001-1003

S 0002-9939(97)03650-2

\title{
ON AN OPTIMALITY PROPERTY OF RAMANUJAN SUMS
}

\author{
GENNADY BACHMAN
}

(Communicated by William W. Adams)

\begin{abstract}
We evaluate $\inf _{b_{n}} \sum_{a=1}^{q}\left|\sum_{n=1}^{q} b_{n} e^{2 \pi i a n / q}\right|$, where the inf is taken over sequences $b_{n}$ satisfying $b_{n} \geq 1$. In particular we show that it is attained by taking $b_{n}=1$ for all $n$, which reduces the summation over $n$ to a Ramanujan sum $c_{q}(a)=\sum_{\substack{n=1 \\(n, q)=1}}^{q} e^{2 \pi i a n / q}$.
\end{abstract}

Let a positive integer $q$ be fixed. In this note we consider the problem of determining

$$
\inf _{b_{n}} \sum_{a=1}^{q}\left|\sum_{n}^{\prime} b_{n} e\left(\frac{a n}{q}\right)\right|
$$

where $e(\alpha)$ stands for $e^{2 \pi i \alpha}, \sum_{n}^{\prime}$ denotes the summation over $n$ in the range $1 \leq$ $n \leq q,(n, q)=1$, and the infimum is taken over all sequences $b_{n}$ satisfying

$$
b_{n} \geq 1 \text {. }
$$

We note that if $b_{n}=1$ for all $n$, the innermost sum in (1) is the Ramanujan sum

$$
c_{q}(a)=\sum_{n}^{\prime} e\left(\frac{a n}{q}\right) .
$$

Using the well-known identity

$$
c_{q}(a)=\frac{\varphi(q) \mu(q /(a, q))}{\varphi(q /(a, q))},
$$

where $\varphi$ and $\mu$ are Euler's and Möbius functions respectively (see, for example, [HW, Theorem 272]), and letting $q_{0}=\prod_{p \mid q} p$ be the square-free kernel of $q$, we obtain

$$
\begin{aligned}
\sum_{a=1}^{q}\left|c_{q}(a)\right| & =\sum_{a=1}^{q} \frac{\varphi(q) \mu^{2}(q /(a, q))}{\varphi(q /(a, q))} \\
& =\varphi(q) \sum_{d \mid q_{0}} \frac{1}{\varphi(d)} \sum_{\substack{1 \leq a \leq q \\
(a, q)=q / d}} 1=\varphi(q) \sum_{d \mid q_{0}} 1 \\
& =\varphi(q) 2^{\omega(q)},
\end{aligned}
$$

where $\omega(q)$ is the number of distinct prime divisors of $q$. Thus (4) gives a "trivial" upper bound for (1). In fact this author was led to consider this problem while

Received by the editors March 28, 1995 and, in revised form, October 19, 1995.

1991 Mathematics Subject Classification. Primary 11L03. 
working on estimates for more general exponential sums in an attempt to beat this estimate by introducing a "smoothing factor" $b_{n}$ which had to satisfy (2). This might seem plausible at first since if $q /(a, q)>3$, one can easily find $b_{n}$ satisfying (2) for which

$$
\sum_{n}^{\prime} b_{n} e\left(\frac{a n}{q}\right)=0 .
$$

We will show however that (1) is attained by taking $b_{n}=1$ for all $n$. In fact, the following more general result is no more difficult.

Theorem. Let $r$ be a real number satisfying $r \geq 1$. Then for any sequence of complex numbers $b_{n}$ we have

$$
\sum_{a=1}^{q}\left|\sum_{n}^{\prime} b_{n} e\left(\frac{a n}{q}\right)\right|^{r} \geq\left(\frac{\left|\sum_{n}^{\prime} b_{n}\right|}{\varphi(q)}\right)^{r} \sum_{a=1}^{q}\left|c_{q}(a)\right|^{r} .
$$

Proof. We may assume that $b_{n}$ is defined for all integers $n$ and is periodic with period $q$. We set

$$
B=\sum_{n}^{\prime} b_{n}
$$

and

$$
S=\sum_{a=1}^{q}\left|\sum_{n}^{\prime} b_{n} e\left(\frac{a n}{q}\right)\right|^{r} .
$$

Using $n^{*}$ to denote the multiplicative inverse of $n$ modulo $q$ for $(n, q)=1$, we write

$$
\begin{aligned}
|B|^{r} \sum_{a=1}^{q}\left|c_{q}(a)\right|^{r} & =\sum_{a=1}^{q}\left|B c_{q}(a)\right|^{r} \\
& =\sum_{a=1}^{q}\left|\sum_{m}^{\prime}\left(\sum_{n}^{\prime} b_{m n}\right) e\left(\frac{a m}{q}\right)\right|^{r} \\
& =\sum_{a=1}^{q}\left|\sum_{n}^{\prime} \sum_{m}^{\prime} b_{m n} e\left(\frac{a n^{*} m n}{q}\right)\right|^{r} .
\end{aligned}
$$

By the Hölder inequality, or trivially in the case $r=1$, the last summation over $n$ is bounded by

$$
\leq \varphi(q)^{1 / r^{\prime}}\left(\sum_{n}^{\prime}\left|\sum_{m}^{\prime} b_{m n} e\left(\frac{a n^{*} m n}{q}\right)\right|^{r}\right)^{1 / r},
$$

where $r^{\prime}$ satisfies $1 / r+1 / r^{\prime}=1$. Therefore

$$
\begin{aligned}
|B|^{r} \sum_{a=1}^{q}\left|c_{q}(a)\right|^{r} & \leq \varphi(q)^{r / r^{\prime}} \sum_{n}^{\prime} \sum_{a=1}^{q}\left|\sum_{m}^{\prime} b_{m n} e\left(\frac{a n^{*} m n}{q}\right)\right|^{r} \\
& =\varphi(q)^{r / r^{\prime}+1} S \\
& =\varphi(q)^{r} S
\end{aligned}
$$

and the theorem follows. 
We observe that (5) may fail for $r<1$. For example, taking $q=5, b_{1}=b_{4}=$ $1+1 /(2 \cos (2 \pi / 5))$ and $b_{2}=b_{3}=1$, we obtain, by $(3)$,

(6)

$$
\begin{aligned}
\sum_{a=1}^{5}\left|\sum_{n=1}^{4} b_{n} e\left(\frac{a n}{5}\right)\right|^{r} & =\sum_{a=1}^{4}\left|\frac{1}{2 \cos (2 \pi / 5)}\left(e\left(\frac{a}{5}\right)+e\left(\frac{4 a}{5}\right)\right)-1\right|^{r}+\left|4+\frac{1}{\cos (2 \pi / 5)}\right|^{r} \\
& =2\left|\frac{\cos (4 \pi / 5)}{\cos (2 \pi / 5)}-1\right|^{r}+\left|4+\frac{1}{\cos (2 \pi / 5)}\right|^{r},
\end{aligned}
$$

and

$$
\sum_{a=1}^{5}\left|c_{q}(a)\right|^{r}=4+4^{r} .
$$

Thus for $r>0$ sufficiently small (6) will be smaller than (7).

\section{REFERENCES}

[HW] G.H. Hardy \& E.M. Wright, An introduction to the theory of numbers, fifth edition, Oxford University Press, 1979. MR 81i:10002

Department of Mathematical Sciences, University of Nevada, Las Vegas, Nevada 89154-4020

E-mail address: bachman@nevada.edu 